\title{
Caracterização de Isolados de Phytophthora Patogênicos a Citrus sinensis no Estado de Alagoas
}

\author{
Maria de Fátima S. Muniz ${ }^{1}$, Fátima M. Queiroz² \& Maria Menezes ${ }^{3}$ \\ ${ }^{1}$ Depto. de Fitotecnia e Fitossanidade, Centro de Ciências Agrárias,Universidade Federal de Alagoas, CEP 57100-000, Rio \\ Largo, AL; ${ }^{2}$ Diretoria de Pesquisa Agropecuária e Pesqueira, Secretaria Executiva de Agricultura, Irrigação, Pesca e \\ Abastecimento, CEP 57020-050, Maceió, AL; ${ }^{3}$ Setor de Fitossanidade, Depto. de Agronomia, Universidade Federal Rural de \\ Pernambuco, CEP 52171-900, Recife, PE
}

(Aceito para publicação em 06/01/2004)

Autor para correspondência: Maria de Fátima S. Muniz

MUNIZ, M.F.S., QUEIROZ, F.M. \& MENEZES, M. Caracterização de isolados de Phytophthora patogênicos a Citrus sinensis no Estado de Alagoas. Fitopatologia Brasileira 29:201-204. 2004.

\section{RESUMO}

Nove isolados de Phytophthora obtidos da rizosfera de laranjeiras (Citrus sinensis) infetadas, em Alagoas, foram caracterizados com base na morfologia da colônia, morfologia e morfometria das estruturas reprodutivas, e no crescimento em diferentes temperaturas. Todas as culturas apresentaram-se heterotálicas; esporângios papilados, ovóides ou subesféricos, medindo 24,6 - 78,7 $\mu \mathrm{m}$ de comprimento (média=49,1 $\mu \mathrm{m}$ ) x 16,4 - 49,2 $\mu \mathrm{m}$ de largura (média $=33,3 \mu \mathrm{m}$ ), com uma relação comprimento/largura de 1,1 - 2,3 (média=1,5); clamidósporos principalmente terminais, apresentando 13,1 - 45,9 $\mu \mathrm{m}$ de diâmetro (média=27,3 $\mu \mathrm{m}$ ). Oogônios globosos, com 14,8 - 34,4 $\mu \mathrm{m}$ de diâmetro (média=26,4 $\mu \mathrm{m}$ ) contendo oósporos apleuróticos, medindo 11,5 - 29,5 $\mu \mathrm{m}$ de diâmetro (média=22,7 $\mu \mathrm{m}$ ). Anterídios em posição anfígena, medindo 6,6 $16,7 \mu \mathrm{m}$ de comprimento (média $=10,6 \mu \mathrm{m}$ ) e 8,2 - 16,7 $\mu \mathrm{m}$ de largura (média $=12,0 \mu \mathrm{m}$ ). O maior crescimento micelial ocorreu entre $25 \mathrm{e}$ $30{ }^{\circ} \mathrm{C}$ em meio de cenoura-ágar modificado. Todos os isolados cresceram a $35{ }^{\circ} \mathrm{C}$ e foram patogênicos às mudas de limão (Citrus limonia) 'Cravo' e aos frutos de laranja 'Pêra'. Todos os isolados foram identificados como $P$. nicotianae $(=P$. parasitica), pertencentes ao tipo compatível A1.

Palavras-chave adicionais: taxonomia, gomose-dePhytophthora, Citrus spp., laranjeiras.

\section{ABSTRACT}

\begin{abstract}
Characterization of Phytophthora isolates pathogenic to Citrus sinensis in the State of Alagoas, Brazil

Nine Phytophthora spp. isolates from the rhizosphere of infected orange (Citrus sinensis) plants in Alagoas were characterized based on colony morphology, morphology and morphometry as well as reproductive structures, and cardinal growth temperatures. All cultures were heterothallic, with papillate, ovoid or subspherical sporangia measuring $24.6-78.7$ $\mu \mathrm{m}$ long x $16.4-49.2 \mu \mathrm{m}$ wide, average 49.1 x $33.3 \mu \mathrm{m}$. The lengthbreadth ratio was $1.1-2.3$, average 1.5 . Chlamydospores were mostly
\end{abstract}

terminal, $13.1-45.9 \mu \mathrm{m}$ in diameter, average $27.3 \mu \mathrm{m}$. Oogonia were globose, $14.8-34.4 \mu \mathrm{m}$ in diameter, average $26.4 \mu \mathrm{m}$ containing aplerotic oospores $11.5-29.5 \mu \mathrm{m}$ in diameter, average $22.7 \mu \mathrm{m}$ and amphigynous antheridia $6.6-16.7 \mu \mathrm{m} \times 8.2-16.7 \mu \mathrm{m}$ (average $10.6 \mu \mathrm{m} \times 12.0 \mu \mathrm{m}$ ). The greatest mycelial growth was observed at $25-30{ }^{\circ} \mathrm{C}$ in modified carrot agar medium. All isolates grew at $35{ }^{\circ} \mathrm{C}$ and were pathogenic to lemon (Citrus limonia) cv. Cravo seedlings and ripened fruits of orange $(C$. sinensis) $\mathrm{cv}$. Pera. The isolates were identified as Phytophthora nicotianae $(=P$. parasitica) of the A1 mating type.
Entre as espécies de citrus, a cultura da laranja [Citrus sinensis (L.) Osbeck] ocupa praticamente $100 \%$ da área cultivada em Alagoas, sendo a terceira mais importante dentre as frutíferas, com uma área colhida de 3.761 ha, destacandose os municípios de Santana do Mundaú, União dos Palmares e Jacuípe (IBGE, 2000). Entretanto, a produtividade é baixa devido ao uso generalizado de mudas sem boas qualidades genética e sanitária.

O Estado de Alagoas ainda não dispõe de normatização para produção de mudas de citros. Em função disso, os viveiristas produzem mudas em viveiros de campo, instalados na mesma área dos plantios comerciais, contribuindo assim, para a contaminação das mudas por pragas e doenças.

Dentre as doenças que afetam a laranjeira em Alagoas, a gomose causada por Phytophthora spp., é a de maior importância em todos os municípios produtores.

Diferentes espécies tais como $P$. boehmeriae Saw., $P$. cactorum (Lebert \& Cohn) Schroeter, P. capsici Leonian, $P$. cinnamomi Rands, P. citricola Saw., P. citrophthora (Sm. \& Sm.) Leonian, P. drechsleri Tucker, $P$. hibernalis Carne, $P$. megasperma Drechsler, P. nicotianae $(=P$. parasitica) Dastur, P. nicotianae (=P. nicotianae van Breda de Haan var.parasitica (Dastur) Waterhouse), P. palmivora (Butler) Butler e $P$. syringae (Kleb.) Kleb., já foram relatadas como patogênicas à cultura (Feichtenberger et al., 1997; Feichtenberger, 2001). No Brasil, $P$. parasitica e $P$. citrophthora são os mais comuns e danosos agentes etiológicos desta doença (Rossetti, 2001), sendo $P$. parasitica a espécie predominante nas principais 
regiões produtoras (Feichtenberger, 2001).

Os sintomas da infecção são lesões na casca da base da planta, raízes e nos galhos baixos, com exsudação de goma pelo fendilhamento da casca. Pode-se observar ainda, na parte interna da casca, uma coloração pardacenta. Com o progresso da doença, os tecidos apodrecem e quando a lesão envolve toda a circunferência do tronco, a planta morre rapidamente (Davis, 1988; Santos Filho, 1991).

Em Alagoas, não existem dados publicados sobre a etiologia das espécies de Phytophthora patogênicas à laranjeira. Assim sendo, julgou-se pertinente a realização deste trabalho que teve como objetivos: a) identificar morfofisiologicamente isolados de Phytophthora sp. de laranja das cultivares Bahia, Comum, Lima e Pêra, e b) avaliar a patogenicidade de isolados de Phytophthora sp. associados com a gomose da laranjeira.

Este trabalho foi conduzido no Laboratório de Fitopatologia do Centro de Ciências Agrárias (CECA) da Universidade Federal de Alagoas (UFAL), em Rio Largo, Alagoas.

A metodologia empregada para a obtenção dos isolados foi aquela proposta por Matheron \& Matejka (1991). Amostras de solos obtidas da rizosfera de laranjeiras com sintomas de gomose, foram colocadas em béquer $(600 \mathrm{ml})$ acrescentandose água suficiente para deixar uma camada aquosa de 1 a 2 $\mathrm{cm}$. Um fruto maduro de pêra cv. Anjou usado como isca, foi colocado sobre a superfície de cada amostra de solo, por período de dois a cinco dias, à temperatura ambiente (aproximadamente $28^{\circ} \mathrm{C}$ ). As iscas apresentando lesões foram removidas do solo e lavadas em água corrente, procedendose o isolamento, que consistiu na retirada de pequenos fragmentos da região de transição entre o tecido sadio e o infetado. Após a desinfestação superficial com hipoclorito de sódio a $1 \%$, os fragmentos foram transferidos para placas de Petri contendo $15 \mathrm{ml}$ do meio batata-dextrose-ágar (BDA) adicionado de benomil, nistatina, PCNB, rifampicina e ampicilina (Masago et al., 1977). As placas foram incubadas no escuro, à temperatura ambiente, e as colônias que se desenvolveram após 24-96 h, foram transferidas para placas de Petri contendo meio de BDA e, posteriormente, preservadas em água destilada esterilizada (Castellani, 1967) e mantidas à temperatura ambiente.

$\mathrm{Na}$ Tabela 1 encontram-se caracterizados os isolados de Phytophthora sp. obtidos de diferentes municípios do Estado de Alagoas.

Para identificação dos isolados, discos de micélio (5 mm de diâmetro), retirados das margens de colônias de cada isolado, em BDA, a $25^{\circ} \mathrm{C}$, no escuro, foram transferidos para o centro de placas de Petri contendo $15 \mathrm{ml}$ do meio cenouraágar modificado - CA (Ribeiro, 1978). Em seguida, as placas foram mantidas a $10,15,20,25,30$ e $35^{\circ} \mathrm{C}$, em escuro contínuo, e cinco dias após foi medido o diâmetro médio das colônias, como também observado o aspecto cultural. O delineamento experimental foi o inteiramente casualizado em esquema fatorial (isolados $\mathrm{x}$ temperaturas) com três repetições, de uma placa de Petri cada.
Também foi determinada a produção, forma e dimensão das estruturas assexuais produzidas por Phytophthora sp., procedendo-se da seguinte forma: discos de micélio $(5 \mathrm{~mm}$ de diâmetro), retirados das bordas de colônias de cada isolado, em meio CA, incubados a $25^{\circ} \mathrm{C}$, em regime alternado de luz e escuro durante três dias, foram transferidos para solução de $\mathrm{KNO}_{3}$ 0,01 M (Erwin \& Ribeiro, 1996). Após dois dias, nas mesmas condições de incubação, foram efetuadas as avaliações das estruturas.

A formação dos oósporos foi avaliada por meio do pareamento dos isolados testados, com os isolados de P. capsici (59 e 46), pertencentes aos grupos de compatibilidade A1 e A2 respectivamente, fornecidos pela micoteca do CEPEC/ CEPLAC, Itabuna, BA. O método de pareamento usado foi do tipo sanduíche conforme descrito por Kellam \& Zentmyer (1986). Discos de micélio (7 mm de diâmetro), retirados das margens de colônias de cada isolado a ser testado, e outro, contendo micélio de A1 ou A2 ou do próprio isolado foram pareados, em meio CA, intercalados por um disco do referido meio de cultura. Em seguida, as placas foram seladas com filme de PVC e as culturas incubadas a $20^{\circ} \mathrm{C}$, no escuro. Após sete dias, foram observados oósporos de Phytophthora sp., nos discos de meio CA que foram intercalados. Cada isolado foi representado por três repetições, consistindo cada repetição de um pareamento.

As medições das estruturas propagativas foram realizadas, com auxílio de microscópio equipado com micrômetro ocular. Para isso, foram efetuadas 50 medições de cada tipo de estrutura propagativa por isolado.

Para a determinação e caracterização de Phytophthora sp. foi utilizada a chave de identificação proposta por Erwin \& Ribeiro (1996).

A patogenicidade foi testada, em mudas do portaenxerto limão 'Cravo' (Citrus limonia Osbeck) com cinco meses de idade, produzidas em copos plásticos $(400 \mathrm{ml})$, contendo solo esterilizado. Quatro mudas foram utilizadas para inoculação com cada isolado. Discos de micélio $(5 \mathrm{~mm}$ de diâmetro) retirados das margens de colônias de cada isolado,

TABELA 1 - Identidade e procedência dos isolados de Phytophthora sp. obtidos da rizosfera de laranjeiras (Citrus sinensis) infetadas em Alagoas

\begin{tabular}{cll}
\hline \hline Identificação & Cultivar & Município \\
\hline P15 & Bahia & Jacuípe \\
P16 & Comum & Jacuípe \\
P17 & Comum & Jacuípe \\
P20 & Lima & Jacuípe \\
P22 & Pêra & Jacuípe \\
P24 & Comum & Água Branca \\
P35 & Bahia & União dos Palmares \\
P39 & Lima & Santana do Mundaú \\
P43 & Lima & Santana do Mundaú \\
\hline
\end{tabular}


Caracterização de isolados de Phytophthora patogênicos a Citrus sinensis...

foram inseridos em ferimentos realizados com auxílio de bisturi, localizados no colo das plantas, com aproximadamente $5 \mathrm{~mm}$ de extensão. Em seguida, a região inoculada foi envolvida com filme de PVC. Nas mudas que serviram de testemunhas, foi inserido no colo apenas um disco de BDA, sem o inóculo. Após a inoculação, os copos contendo as mudas foram mantidos em bandejas com água, no laboratório, à temperatura ambiente. Aos 90 dias da inoculação das plantas, observaram-se os sintomas e procedeu-se ao reisolamento do patógeno em meio seletivo, conforme descrito anteriormente.

A patogenicidade também foi testada em frutos maduros de laranja cv. Pêra, os quais foram lavados em água corrente, imersos em solução de hipoclorito de sódio $2 \%$, durante $5 \mathrm{~min}$ e, em seguida, lavados em água destilada esterilizada e secos com papel-toalha. Seis frutos de laranja foram utilizados para inoculação com cada isolado de Phytophthora sp.

A inoculação foi efetuada em pequenos ferimentos realizados com estilete na parte mediana de cada fruto, depositando-se no local, um disco de micélio $(5 \mathrm{~mm}$ de diâmetro), retirado das margens de colônias de cada isolado, e após, fixado por uma fita adesiva. Frutos testemunhas receberam procedimento semelhante, utilizando-se apenas discos do meio de cultura, sem o inóculo.

Os frutos inoculados e as testemunhas foram mantidos em sacos plásticos contendo algodão umedecido com água esterilizada, e incubados à temperatura de $26 ? 1^{\circ} \mathrm{C}$, durante sete dias.

A avaliação foi realizada pela observação de frutos com sintomas de apodrecimento, efetuando-se também o reisolamento do microrganismo em meio seletivo, conforme descrito anteriormente.

Todos os isolados foram obtidos de solo, pela técnica de isca (Tabela 1). Isolamentos feitos a partir dos tecidos infetados das plantas de laranja não resultaram em culturas puras. De acordo com Erwin \& Ribeiro (1996), as razões para esta dificuldade podem ser atribuídas ao efeito supressivo de bactérias antagônicas e/ou à ausência de micélio viável.

Houve variação quanto ao crescimento micelial dos isolados de Phytophthora sp. nas diferentes temperaturas, tanto entre as temperaturas como entre os isolados (Figura 1). Todos os isolados cresceram a $35^{\circ} \mathrm{C}$, porém, o maior diâmetro das colônias ocorreu entre 25 e $30^{\circ} \mathrm{C}$. Nenhum dos isolados cresceu a $10^{\circ} \mathrm{C}$. De acordo com Holliday (1980), a temperatura ótima para o crescimento micelial de $P$. nicotianae é 25 a 30 ${ }^{\circ} \mathrm{C}$, estando de acordo com o encontrado em nossos estudos para os isolados de Phytophthora sp. de solo de laranjeira. No meio CA, as colônias apresentaram aspecto cotonoso.

Os esporângios dos nove isolados apresentaram-se ovóides ou subesféricos, papilados, ocasionalmente bipapilados, com comprimento de $24,6-78,7$ ? $\mathrm{m}$ (média=49,1? $\mathrm{m}$ ) e largura de $16,4-49,2$ ?m (média=33,3 ? m). A relação comprimento/ largura variou de 1,1 - 2,3 (média=1,5) (Tabela 2).

Os clamidósporos foram predominantemente terminais com o diâmetro variando de 13,1 - 45,9 $\mu \mathrm{m}$ (média=27,3 $\mu \mathrm{m}$ ). Os oogônios apresentaram-se globosos com diâmetro de 14,8
- 34,4 $\mu \mathrm{m}$ (média=26,4 $\mu \mathrm{m}$ ), contendo oósporos apleuróticos, medindo 11,5 - 29,5 $\mu \mathrm{m}$ de diâmetro (média $=22,7 \mu \mathrm{m}$ ); anterídios em posição anfígena, com 6,6 - 16,7 $\mu \mathrm{m}$ de comprimento (média=10,6 $\mu \mathrm{m}$ ) e 8,2 - 16,7 $\mu \mathrm{m}$ de largura (média=12,0 $\mu \mathrm{m}$ ) (Tabela 2). Não houve formação de oogônios e anterídios em monocultura, tratando-se, portanto, de culturas heterotálicas. Todos os isolados testados produziram oogônios e anterídios com o padrão A2, pertencendo ao tipo compatível A1.

As médias dos esporângios, da relação $\mathrm{C} / \mathrm{L}$, dos clamidósporos, dos oogônios e oósporos de cada isolado estão de acordo com os intervalos apresentados por Erwin \& Ribeiro (1996), em relação a $P$. nicotianae (=P. parasitica). Entretanto, as médias do comprimento e largura do anterídio não se enquadraram neste intervalo (Tabela 2). Este resultado pode ser atribuído às diferenças de meios de cultura, condições de ambiente, como também a constituição genética dos isolados.

Segundo Feichtenberger (2001), P. nicotianae é a espécie predominante nas principais regiões produtoras. Entretanto, esta afirmação não pode ser comprovada neste trabalho, devido, ao número reduzido de isolados utilizados.

Todos os isolados foram patogênicos quando inoculados em frutos de laranja 'Pêra', entretanto, os isolados P16 e P20 não foram patogênicos em mudas de limão 'Cravo'. Segundo Luz \& Matsuoka (1996) as espécies de Phytophthora, em sua maioria, são fitopatogênicas.

Plantas inoculadas com os isolados de P. nicotianae produziram apenas lesões necróticas no caule. Não se observou clorose nas folhas ou morte de plantas aos 90 dias após a inoculação. Plantas nas quais se depositou apenas disco de meio de cultura não desenvolveram sintomas.

Com relação aos frutos inoculados com $P$. nicotianae os sintomas apareceram dois dias após, iniciando com lesões encharcadas, levemente descoloridas, evoluindo para coloração marrom e consistência mole, aos sete dias de observação, culminando com o apodrecimento total do fruto. Sobre o tecido lesionado foi evidenciado a presença de micélio branco, cotonoso.

Culturas reisoladas a partir dos tecidos afetados das

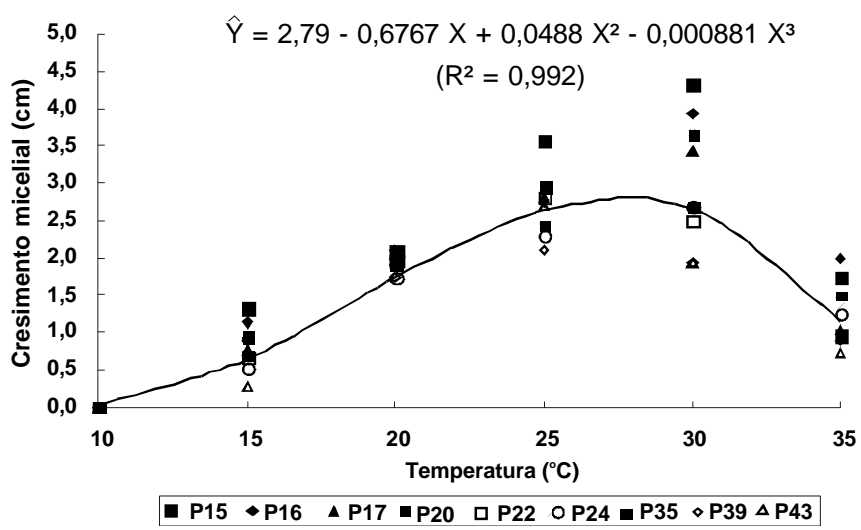

FIG. 1 - Crescimento micelial (cm) de nove isolados de Phytophtora sp. de laranjeira (Citrus sinensis), a 10, 15, 20, 25, 30 e $35^{\circ} \mathrm{C}$. 
M.F.S. Muniz et al.

TABELA 2 - Dimensões médias das estruturas propagativas dos isolados de Phytophthora sp. obtidos da rizosfera de laranjeiras (Citrus sinensis.) infetadas no Estado de Alagoas

\begin{tabular}{|c|c|c|c|c|c|c|c|c|}
\hline \multirow{2}{*}{ Isolado } & \multicolumn{3}{|c|}{$\begin{array}{c}\text { Esporângio } \\
\text { Dimensões (? m) }{ }^{1}\end{array}$} & \multirow{2}{*}{$\begin{array}{l}\text { Diâmetro }(? \mathbf{m})^{1} \\
\text { Clamidósporo }\end{array}$} & \multicolumn{2}{|c|}{ Diâmetro (? m) ${ }^{1}$} & \multicolumn{2}{|c|}{$\begin{array}{c}\text { Anterídio } \\
\text { Dimensões (? m) }\end{array}$} \\
\hline & $\begin{array}{l}\text { Comp. } \\
\text { (C) }\end{array}$ & $\begin{array}{c}\text { Larg. } \\
(\mathrm{L})\end{array}$ & $\mathrm{C} / \mathrm{L}$ & & Oogônio & Oósporo & $\begin{array}{l}\text { Comp. } \\
\text { (C) }\end{array}$ & $\begin{array}{c}\text { Larg. } \\
\text { (L) }\end{array}$ \\
\hline P15 & 54,1 & 36,8 & 1,5 & 26,7 & 28,5 & 24,7 & 9,6 & 12,3 \\
\hline P16 & 42,5 & 28,7 & 1,5 & 23,6 & 26,2 & 22,7 & 12,4 & 10,5 \\
\hline P17 & 52,8 & 34,9 & 1,5 & 26,9 & 26,0 & 22,7 & 9,6 & 12,1 \\
\hline $\mathrm{P} 20$ & 50,6 & 36,0 & 1,4 & 25,7 & 24,7 & 20,6 & 10,5 & 12,3 \\
\hline $\mathrm{P} 22$ & 44,9 & 30,4 & 1,5 & 24,4 & 24,6 & 20,7 & 9,7 & 11,7 \\
\hline $\mathrm{P} 24$ & 47,9 & 34,4 & 1,4 & 34,4 & 27,5 & 23,7 & 11,1 & 13,5 \\
\hline P35 & 51,4 & 37,2 & 1,4 & 36,8 & 26,1 & 22,4 & 10,8 & 12,2 \\
\hline P39 & 48,4 & 30,6 & 1,6 & 24,8 & 26,1 & 21,7 & 10,1 & 11,6 \\
\hline $\mathrm{P} 43$ & 49,7 & 30,4 & 1,6 & 22,0 & 28,3 & 24,9 & 11,7 & 11,4 \\
\hline Média & 49,1 & 33,3 & 1,5 & 27,3 & 26,4 & 22,7 & 10,6 & 12,0 \\
\hline Desvio padrão & 3,70 & 3,24 & 0,08 & 5,00 & 1,40 & 1,55 & 0,99 & 0,82 \\
\hline C.V $(\%)$ & 7,5 & 9,7 & 5,3 & 18,4 & 5,3 & 6,9 & 9,3 & 6,8 \\
\hline $\mathrm{I}^{2}$ & $11-60$ & $20-45$ & $1,1-1,7$ & $13-60$ & $15-64$ & $13-35$ & $10-12$ & $9-10$ \\
\hline
\end{tabular}

${ }^{1}$ Médias de 50 medições de cada estrutura propagativa/isolado.

${ }^{2}$ Intervalos dados para a espécie pela chave de identificação.

plantas e frutos foram semelhantes àquelas utilizadas para inoculação, confirmando-se os postulados de Koch.

\section{AGRADECIMENTOS}

À Fundação de Amparo à Pesquisa do Estado de Alagoas (FAPEAL), por ter financiado este trabalho.

Ao Prof. Gilson Moura Filho, do CECA/UFAL, pela colaboração na análise estatística.

\section{REFERÊNCIAS BIBLIOGRÁFICAS}

CASTELLANI, A. Maintenance and cultivation of the common pathogenic fungi of man in sterile distilled water. Further researches. Journal of Tropical Medicine and Hygiene 70:181-184. 1967.

DAVIS, R.M. Phytophthora-Induced diseases. In: Whiteside, J.O., Garnsey, S.M. \& Timmer, L.W. (Eds.). Compendium of Citrus Diseases. St. Paul. APS. 1988. pp.22-24.

ERWIN, D.C. \& RIBEIRO, O.K. Phytophthora Diseases Worldwide. St. Paul, Minnesota. APS Press.1996.

FEICHTENBERGER, E., MUlLER, G.W. \& GUIRADO, N. Doenças dos citros (Citrus spp.). In: Kimati, H., Amorim, L., Bergamin Filho, A., Camargo, L.E.A. \& Rezende, J.A.M. (Eds.) Manual de Fitopatologia. Doenças das plantas cultivadas. São Paulo. Agronômica Ceres. 1997. v.2, 3.ed., pp.261-297.

FEICHTENBERGER, E. Doenças incitadas por Phytophthora em citros. In: Luz, E.D.M.N., Santos, A.F., Matusoka, K. \& Bezerra,
J.L. (Eds.). Doenças Causadas por Phytophthora no Brasil. Campinas. Livraria e Editora Rural. 2001. pp.283-342.

HOLLIDAY, P. Fungus Diseases of Tropical Crops. Cambridge. Cambridge University Press. 1980.

IBGE. Sistema IBGE de recuperação automática. Rio de Janeiro: Instituto Brasileiro de Geografia e Estatística, 2000. Disponível em <http://www.sidra.ibge.gov.br >Acesso em: 24 de agosto de 2002.

KELLAM, M.K. \& ZENTMYER, G.A.. Morphological, physiological, ecological, and pathological comparisons of Phytophthora species isolated from Theobroma cacao. Phytopathology 76:159-164. 1986.

LUZ, E.D.M.N. \& MATSUOKA, K. Taxionomia e sistemática do gênero Phytophthora. In: Luz, W.C. (Ed.). Revisão Anual de Patologia de Plantas. Passo Fundo. Embrapa Trigo. 1996. v.4. pp.297-328.

MASAGO, H., YOSHIKAWA, M., FUKADA, M. \& NAKANISH, N. Selective inhibition of Pythium spp. on a medium for direct isolation of Phytophthora spp. from soils and plants. Phytopathology 67:425-428. 1977.

MATHERON, M.E. \& MATEJKA, J.C. Effect of sodium tetrathiocarbonate, metalaxyl and fosetyl - Al on development and control of Phytophthora root rot of citrus. Plant Disease 75:264268. 1991.

RIBEIRO, O.K. A Source Book of the Genus Phytophthora. Vaduz. J. Cramer. 1978.

ROSSETTI, V.V. Manual Ilustrado de Doença dos Citros. Piracicaba. Fealq/Fundecitros. 2001.

SANTOS FILHO, H.P. Gomose dos citros. Cruz das Almas, BA. EMBRAPA-CNPMF, 2p. Citros em Foco, 15. 1991 